\title{
COMT Val158Met Polymorphism, Executive Dysfunction, and Sexual Risk Behavior in the Context of HIV Infection and Methamphetamine Dependence
}

\author{
C. A. Bousman, ${ }^{1,2}$ M. Cherner, ${ }^{1}$ J. H. Atkinson, ${ }^{1}$ R. K. Heaton, ${ }^{1}$ I. Grant, ${ }^{1}$ \\ I. P. Everall, ${ }^{1}$ and The HNRC Group ${ }^{3,4}$ \\ ${ }^{1}$ Department of Psychiatry, University of California San Diego, La Jolla, CA 92103, USA \\ ${ }^{2}$ San Diego Joint Doctoral Program in Public Health (Health Behavior), San Diego State/University of California, CA 92182, USA \\ ${ }^{3}$ The Naval Hospital, University of California, San Diego, CA 92055, USA \\ ${ }^{4}$ The Veterans Affairs San Diego Healthcare System, CA 92161, USA
}

Correspondence should be addressed to C. A. Bousman, cbousman@ucsd.edu

Received 18 August 2009; Accepted 10 October 2009

Academic Editor: Marylou V. Solbrig

Copyright (C) 2010 C. A. Bousman et al. This is an open access article distributed under the Creative Commons Attribution License, which permits unrestricted use, distribution, and reproduction in any medium, provided the original work is properly cited.

\begin{abstract}
Catechol-O-methyltransferease (COMT) metabolizes prefrontal cortex dopamine (DA), a neurotransmitter involved in executive behavior; the Val158Met genotype has been linked to executive dysfunction, which might increase sexual risk behaviors favoring HIV transmission. Main and interaction effects of COMT genotype and executive functioning on sexual risk behavior were examined. 192 sexually active nonmonogamous men completed a sexual behavior questionnaire, executive functioning tests, and were genotyped using blood-derived DNA. Main effects for executive dysfunction but not COMT on number of sexual partners were observed. A COMT x executive dysfunction interaction was found for number of sexual partners and insertive anal sex, significant for carriers of the Met/Met and to a lesser extent Val/Met genotypes but not Val/Val carriers. In the context of HIV and methamphetamine dependence, dopaminergic overactivity in prefrontal cortex conferred by the Met/Met genotype appears to result in a liability for executive dysfunction and potentially associated risky sexual behavior.
\end{abstract}

\section{Introduction}

HIV infection is a global pandemic and the population is growing due to successful treatment with highly active antiretroviral therapy (HAART) [1]. Although rates of HIV have been reduced in the United States among most groups as a result of successful public health efforts (e.g., condom accessibility, education programs, media campaigns), sexual risk behavior and subsequent acquisition and/or spread of HIV and other sexually transmitted infections are still of concern among men who have sex with men as well as drug using populations [1]. Thus, it is evident that, despite research and efforts to understand and curb sexual risk behavior within these vulnerable populations, additional work employing novel approaches are needed.

Sexual risk behaviors can be viewed as a composite of numerous behaviors that collectively make-up a complex behavioral phenotype. As with most complex phenotypes, sexual risk behavior is heterogeneous and several factors contribute to the variance that can be observed from one individual to another. To date, a majority of work examining risk factors for sexual risk behavior phenotypes have primarily focused on psychosocial factors (reviewed in [2]) and/or other complex/heterogeneous behavioral phenotypes such as substance use behaviors $[3,4]$ as indicators for current or future sexual risk behavior. Ultimately these indicators, upon sufficient replication, become candidates for public health interventions that aim to prevent and reduce sexual risk behaviors. However, the trouble with many of these candidates is that they are too proximal to sexual risk behaviors and often cooccur, making it difficult to disentangle temporal precedence and ultimately limit prevention efforts. One relatively novel approach is to examine intermediate phenotypes or endophenotypes [5] such as neurocognitive 
factors as well as biological (i.e., genetic) factors. These factors are more distal to the onset of sexual risk behavior and thus are potentially more advantageous candidates for identifying vulnerable individuals and informing prevention efforts for sexual risk behavior.

Studies in literature examining neurocognitive and biological factors as indicators for sexual risk behaviors are limited. In fact, only two studies to date have examined neurocognitive factors $[6,7]$ and none to our knowledge have examined biological factors as potential indicators. Although this paucity of research is surprising given previous work linking both neurocognitive [8-10] and genetic [11-13] indicators to other health related behaviors, research has established the dopminergic system as a common link between neurocognitive functioning and sexual behavior.

The dopminergic system has been shown to be involved in sexual arousal, motivation and the subsequent rewarding effect of sexual behavior (for detailed review see [14]). Furthermore, DA in the human brain, specifically in the prefrontal cortex (PFC), has been shown to be necessary for proper cognitive functioning to occur and high or low levels of DA in this brain region are known to contribute to individual cognitive differences in humans $[15,16]$. The PFC is of particular importance when examining risk behavior in that executive functions such as decision-making, planning, self-monitoring as well as behavior initiation, organization, and inhibition are largely dependent on PFC integrity [17]. Impairment in executive functioning may result in difficulties in assessing relationships between a person's current behavior and future outcomes; thereby resulting in choices and/or responses on the premise of immediate rewards (e.g., pleasure, social acceptance) versus longterm consequences (e.g., viral infections) and an ultimate potential increase in the likelihood for participation in sexual risk behaviors (e.g., unprotected sex, multiple partners) $[7,18]$. Thus, mechanisms responsible for maintaining a dopamine balance within the brain and in particular the PFC would appear to be good biological candidates for further exploration of an association between executive dysfunction and sexual risk behavior.

One such candidate is catechol-O-methyltransferease $(C O M T)$ which is a mammalian enzyme involved in the metabolic degradation of released dopamine, particularly in the PFC [19]. Of particular interest to this study is a common polymorphism involving a Val to Met substitution at codon 158. The Val allele of the COMT Val158Met polymorphism is $40 \%$ more enzymatically active than the Met allele [20]. Thus, carriers of the Met allele metabolize dopamine at a less efficient rate, resulting in higher levels of dopamine in the synapse and ultimately an escalation in dopamine receptor activation. This differentiation of dopamine receptor activity dependent on COMT genotype has led to several investigations into the relationship between COMT and executive dysfunction in which the Val allele has been putatively linked to poor performance on executive functioning tasks [21]. However, to our knowledge no work has examined the relationship between COMT and sexual risk behavior; albeit studies of similar behaviors such as novelty seeking [22-24], reward dependence [22], as well as affective arousal and regulation [25] have demonstrated significant relationships.

Given the aforementioned paucity of research in the current literature addressing the contribution of genetic and neurocognitive factors on sexual risk behavior, the primary aim of this study was to examine the main effects of executive functioning as well as the main effects of the COMT Val158Met polymorphism on sexual risk behavior among a ethnically diverse population of men with and without METH dependence and/or HIV infection. Within this aim, we hypothesized that the highly active COMT $\mathrm{Val} /$ Val genotype and its putatively associated deficits in executive functioning would be independently associated with sexual risk behaviors. In addition, as a result of previously mentioned research that has demonstrated an association between COMT genotype and executive functioning we also explored the potential interaction effects of COMT and executive dysfunction on sexual risk behavior.

\section{Methods}

2.1. Participants. Participants were volunteers evaluated at the HIV Neurobehavioral Research Center (HNRC) at the University of California in San Diego as part of a cohort study focused on central nervous system effects of HIV and methamphetamine. The current study comprised 192 sexually active non-monogamous men with and without methamphetamine dependence $(\mathrm{METH}+\mathrm{l}-$ ) and/or HIV infection (HIV+/-). Men were classified as nonmonogamous if they stated they had "no current partner" at time of assessment. Monogamous men were excluded because unsafe sexual behavior within a monogamous relationship is less risky than in non-monogamous relationships [26].

All participants underwent a comprehensive characterization procedure that included collection of demographic, neuromedical, psychiatric as well as neuropsychiatric information. HIV serological status was determined by enzyme linked immunosorbent assays (ELISA) plus a confirmatory test. Lifetime METH dependence was determined by the Structured Clinical Interview for the Diagnostic and Statistical Manual of Mental Disorders Version IV (SCIDIV). However, participants were not actively using other substances, with the exception of cannabis and alcohol. Potential participants were excluded if they met lifetime dependence criteria for other drugs, unless the dependence was judged to be remote (more than 5 years ago) and episodic in nature by a doctoral level clinician. Alcohol dependence within the last year was also an exclusion criterion. All participants were seronegative for hepatitis $\mathrm{C}$ infection.

Additional information for each participant was collected as it relates to current depressed mood as well as lifetime diagnosis of Major Depression Disorder (MDD) and/or Bipolar Disorder I or II. Current depressed mood was assessed utilizing the Beck Depression Inventory-I (BDI-I) [27] and MDD and Bipolar Disorder were ascertained using the SCID-IV. Information was also collected to determine lifetime dependence on sedatives, cannabis, opioids, cocaine, 
hallucinogens, and alcohol, using the SCID-IV. For METH+ participants, additional information was collected regarding age at first use, years of use, and days since last use of METH; whereas for HIV+ participants, HIV RNA plasma copies was ascertained as part of a larger neuromedical evaluation. All participants gave written consent prior to enrollment and all procedures were approved by the Human Research Protection Program of the University of California, San Diego and San Diego State University.

2.2. Executive Functioning. Executive functioning was determined as part of a larger comprehensive battery of tests covering seven ability domains (Learning, Memory, Attention/Working Memory, Verbal Fluency, Processing Speed, Abstraction/Problem Solving, and Motor Speed). The executive functioning domain deficit score, of particular focus in this study, was made up of (1) perseverative responses on the Wisconsin Card Sorting Test [28]; (2) errors on the Halstead Category Test [29], which measures abstraction and cognitive flexibility; and (3) time to complete the Trail Making Test part B (Trails B) [30], reflecting ability to switch and maintain attention between ongoing sequences. Raw scores for each of these component tests were converted to demographically-adjusted $T$-scores $(M=50$, $\mathrm{SD}=10$ ), including adjustments for age, education, gender, and ethnicity as available for each test. The demographicallyadjusted $T$-scores for each test were then converted into deficit scores, which reflect degree of impairment by setting performances within the normal range at zero with a range from 0 ( $T$-score > 39; no impairment) to 5 ( $T$-score < 20; severe impairment). Finally, the individual deficit scores were averaged to derive the domain deficit score, which reflects the severity of executive functioning deficit. Previous work has demonstrated that deficit scores achieve good diagnostic agreement with classifications made by blind clinical ratings [31, 32]. All neurocognitive testing and scoring was performed by trained psychometrists blinded to participants' genotypes.

2.3. Sexual Risk Behavior. Sexual risk behavior was assessed through an HNRC-developed self-report measure covering the preceding year. Information was gathered with regard to age at first intercourse as well as number of different sex partners. Age at time of first intercourse was coded in years for both male and female partners. However, when two different ages were given for first intercourse, the younger of the two ages was used. In addition, participants were asked to indicate the percentage of time that they used a condom as well as engaged in oral, vaginal, anal (receptive \& insertive) and/or intoxicated sex. Responses were recorded on a 6item, Likert-type scale with a value of $0=0 \%, 1=1 \%-5 \%$, $2=6 \%-25 \%, 3=26 \%-50 \%, 4=51 \%-75 \%$ and $5=76 \%-$ $100 \%$.

2.4. DNA Extraction and Genotyping. DNA was extracted from peripheral blood mononuclear cells stored (three to five years) at $-70^{\circ} \mathrm{C}$ using the QIAamp DNA Mini kit (Qiagen, Valencia, CA; Catalog \#51185). The COMT Val158Met polymorphism (rs4680) was assayed along with eight other SNPs as part of a concurrent genetic association project at the HNRC. A multiplex PCR technique designed using Sequenom SpectroDESIGNER software (version 3.0.0.3) was employed by inputting a sequence containing $100 \mathrm{bp}$ of flanking sequence on either side of the COMT Val158Met polymorphism. The SNP was then grouped into multiplexes so that the extended product would not overlap in mass with any other oligonucleotide present in the reaction mix, and where no primer-primer, primer-product, or nonspecific interactions would occur. The PCR was carried out in 384-well reaction plates in a volume of $5 \mu \mathrm{l}$ using $10 \mathrm{ng}$ genomic or whole-genome amplified (WGA) DNA. All subsequent steps, up until the reaction, were spotted onto the SpectroCHIP and carried out in the same reaction plate. After PCR, any unincorporated dNTPs from the PCR were removed from the reaction by digestion with Shrimp alkaline phosphatase. dNTPs were removed so that they could not play any role in the extension of the oligonucleotide at the SNP site. The extension reaction was then carried out in the presence of the extension oligonucleotide and a termination mix containing mass-modified dideoxynucleotides which extended the oligonucleotide over the SNP site with one base. Before spotting onto the SpectroCHIP, the reaction was cleaned by incubation with a cation-exchange resin which removed any salts present. The extension product was then spotted onto a 384-well spectroCHIP before being flown in the MALDI-TOF mass spectrometer. Data were collected, in real time, using SpectroTYPER Analyzer 3.3.0.15, SpectraAQUIRE 3.3.1.1 and SpectroCALLER 3.3.0.14 (Sequenom) algorithms. All genotyping was performed by an accredited commercial laboratory (Harvard Medical School-Partners Healthcare Center for Genetics and Genomics, Cambridge, MA CLIA no. 22D1005307).

2.5. Statistical Analysis. All statistical tests and procedures were conducted using SPSS 10.0 (SPSS, 2000). Univariate comparisons across the three COMT genotypes (i.e., Val/Val, $\mathrm{Val} / \mathrm{Met}$, Met/Met) were performed using one-way analysis of variance (ANOVA) for continuous and chi-squared tests for categorical variables. In cases, where data violated normality assumptions medians were calculated and nonparametric tests (i.e., Kruskal-Wallis) performed. To examine the main and explore the interaction effects of executive functioning and COMT on sexual risk behaviors, hierarchical multiple linear regressions in accord with Barron and Kenny's approach [33] were conducted for each of the seven sexual risk behaviors (see Section 2.3) under study. Prior to running each analysis, the executive functioning variable was centered and the COMT genotype contrast coded to reduce problems resulting from multicollinearity (Kraemer and Blasey, 2004). In addition, interaction terms were created by multiplying COMT genotype by the centered executive functioning variable. Next, multiple linear regressions were used to examine potential confounders based on univariate genotype comparisons described above. These confounders included: ethnicity, METH status, HIV status and age at first intercourse. We also included BDI scores based on inclusion of this measure in recent work testing a similar hypothesis 
[7]. Results showed that METH status, HIV status, and age at first intercourse accounted for a significant unique variance for all sexual behaviors under investigation $\left(R^{2}\right.$ range: $0.06-0.39, P s<.02)$. Thus to control for these potential confounding effects, the residuals derived from each of the sexual behavior models were used as the dependent variables for all subsequent regression models. The centered executive functioning variable and COMT genotype as well as the new interaction term were then entered as independent variables into seven individual hierarchical multiple regression models using the residuals described above as the dependent variable. For models in which a significant interaction was observed, a final round of regressions were conducted stratified by COMT genotype to determine the nature of the interaction between executive functioning and COMT on the particular sexual risk behavior. Due to the exploratory nature of the interaction analysiswe selected a relaxed alpha threshold alpha $<.10$ to reduce Type II errors, albeit the traditional alpha threshold of .05 was used for all other analyses.

\section{Results}

3.1. Participant Characteristics. Characteristics of the full sample by each of the three COMT genotypes are summarized in Table 1. All three genotype groups were comparable in age, education, sexual behavior, executive functioning, as well as psychiatric and substance dependence histories. However, Val/Val carriers were significantly more likely to identify as African-American $\left(\chi^{2}=17.67, P=.001\right)$, report an earlier age of first intercourse $\left(F_{(2,189)}=3.51, P=.032\right)$, and be seropositive for HIV $\left(\chi^{2}=6.57, P=.038\right)$. Whereas, Met-carriers (i.e., Met/Met or Val/Met) were significantly more likely to identify as Caucasian $\left(\chi^{2}=14.32, P=.001\right)$. Additionally, among METH+ participants Val/Val carriers reported significantly greater total years of METH use $\left(F_{(2,87)}=3.12, P=.050\right)$ compared to their Met-carrying counterparts.

3.2. Main Effects of Executive Functioning and COMT. Table 2 provides standardized multiple linear regression coefficient estimates for main and interaction effects of executive functioning and COMT genotype for each of the seven sexual risk behaviors adjusting for METH status, HIV status, and age at first intercourse. A significant main effect for the executive functioning domain deficit score was observed for number of partners $(\beta=0.21$, $P=.005$ ). Additionally, results from the individual executive functioning tests showed a significant main effect for $T$ scores on the Wisconsin Card Sort and Halstead Category tests in adjusted models of oral sex $(\beta=0.20, P=.009)$ and condom use ( $\beta=-0.16, P=.030)$, respectively. Main effects were not observed for COMT genotype in any of the regression models.

3.3. Interaction Effects of Executive Functioning and COMT. Applying an exploratory cut-off of $P<.10$, significant interactions between the executive functioning domain deficit score and COMT were observed for number of sexual partners $(\beta=0.50, P=.038)$, insertive anal sex $(\beta=0.50$, $P=.046)$, and receptive anal sex $(\beta=0.50, P=.081)$ (Table 2). Subsequent stratified analysis by COMT genotype, revealed that among carriers of the Met/Met $(\beta=0.52$, $P=.001)$ and to a lesser extent Val/Met $(\beta=0.20, P=.048)$ genotype, increases in the executive functioning domain deficit score was significantly associated with increases in the number of sexual partners in the past 12 months. Stratified analysis for insertive and receptive anal sex revealed similar results. Among Met/Met and Val/Met carriers, an increase in executive deficit scores were associated with an increased frequency of insertive (Met/Met: $\beta=0.18$; Val/Met: $\beta=$ 0.11 ) and receptive (Met/Met: $\beta=0.13$; Val/Met: $\beta=$ 0.11 ) anal sex in the past 12 months, albeit not statistically significant.

Results of regression analyses to examine interactions between each of the three individual executive functioning tests and COMT genotype are also shown in Table 2. For the Wisconsin Card Sort Test no interactions were observed. However, for Trails B, significant interactions with COMT were observed for insertive $(\beta=-0.99, P=.015)$ and receptive $(\beta=-0.75, P=.066)$ anal sex, as well as oral sex $(\beta=-0.68, P=.096)$. Stratified regression analysis showed that among carriers of the Met/Met genotype, poor performance on Trails B (i.e., low $T$-scores) was significantly associated with an increased frequency of insertive $(\beta=$ $-0.38, P=.028)$ but not receptive $(\beta=-0.22, P=$ .225) anal sex. Interestingly, among carriers of the Val/Val genotype, $T$-scores on Trails B had a significant positive association with oral sex $(\beta=0.35, P=.013)$. Finally, for the Halstead Category Test, a single interaction with COMT was observed for condom use $(\beta=-1.13, P=.006)$. Among carriers of the Met/Met $(\beta=-0.49, P=.004)$ and to a lesser extent Val/Met $(\beta=-0.19, P=.064)$ genotype, Halstead Category Test $T$-scores were negatively associated with condom use.

\section{Discussion}

To our knowledge this study is the first to examine main effects as well as explore the interaction effects of COMT genotype and executive functioning on sexual risk behavior. Our main findings suggest significant executive dysfunction main effects for number of sexual partners as well as frequency of oral sex and condom use. In addition, results of our exploratory interaction analyses provide evidence that COMT genotype and executive dysfunction interact in models of number of sexual partners, condom use, insertive and receptive anal sex, as well as oral sex. Stratified analyses further suggest that the strength of these associations is dependent on the number of Met alleles the individual was carrying, with the exception of oral sex in which $\mathrm{Val} / \mathrm{Val}$ was the informative genotype.

Our significant executive dysfunction main effects for sexual risk behaviors are discordant with the only other study, to our knowledge, that has examined the association between executive dysfunction and sexual risk behavior [7]. In that study, no association was found between executive 
TABLE 1: Characteristics of full sample by COMT genotype.

\begin{tabular}{|c|c|c|c|c|c|}
\hline & \multicolumn{5}{|c|}{ COMT genotype } \\
\hline & $\begin{array}{l}\text { Full sample } \\
\quad(n=192)\end{array}$ & $\begin{array}{l}\mathrm{Val} / \mathrm{Val} \\
(n=54)\end{array}$ & $\begin{array}{l}\text { Val/Met } \\
(n=103)\end{array}$ & $\begin{array}{l}\text { Met/Met } \\
(n=35)\end{array}$ & \\
\hline Age (years) $M(\mathrm{sd})$ & $37(9)$ & $35(9)$ & $38(9)$ & $39(11)$ & \\
\hline Education (years) $M(\mathrm{sd})$ & $13(2)$ & $13(2)$ & $13(2)$ & $14(2)$ & \\
\hline WRAT4 $M(\mathrm{sd})$ & $100(12)$ & $99(11)$ & $100(12)$ & $104(11)$ & \\
\hline \multicolumn{6}{|l|}{ Ethnicity (row \%) } \\
\hline Caucasian & 71 & 52 & 78 & 83 & $\mathrm{v} / \mathrm{v}<\mathrm{v} / \mathrm{m}, \mathrm{m} / \mathrm{m}^{* *}$ \\
\hline African-American & 15 & 32 & 7 & 11 & $\mathrm{v} / \mathrm{v}>\mathrm{v} / \mathrm{m}, \mathrm{m} / \mathrm{m}^{* *}$ \\
\hline Hispanic & 14 & 17 & 16 & 6 & \\
\hline \multicolumn{6}{|l|}{ Executive Functioning Battery } \\
\hline Wisconsin card sort test $T(\mathrm{sd})$ & $45(14)$ & $47(16)$ & $44(13)$ & $46(13)$ & \\
\hline Trials part B $T(\mathrm{sd})$ & $49(11)$ & $51(12)$ & $47(10)$ & $52(11)$ & \\
\hline Halstead category Test $T(\mathrm{sd})$ & $46(10)$ & $47(10)$ & $44(10)$ & $47(9)$ & \\
\hline Domain deficit score $M$ (sd) & $.55(.69)$ & $.56(.68)$ & $.62(.74)$ & $.35(.47)$ & \\
\hline Executive impairment (\%) & 45 & 46 & 50 & 31 & \\
\hline \multicolumn{6}{|l|}{ Sexual Characteristics/Behavior } \\
\hline Age at first intercourse $M(\mathrm{sd})$ & $15(4)$ & $14(4)$ & $16(4)$ & $17(4)$ & $\mathrm{v} / \mathrm{v}<\mathrm{m} / \mathrm{m}^{*}$ \\
\hline Sexual preference (\% heterosexual) & 33 & 35 & 31 & 38 & \\
\hline Number partners in past 12 mo Median (IQR) & $3(1,10)$ & $4(1,11)$ & $3(1,10)$ & $2(1,5)$ & \\
\hline Condom use ( $>0 \%$ in past $12 \mathrm{mo})$ & 72 & 74 & 71 & 70 & \\
\hline Insertive anal ( $>0 \%$ in past $12 \mathrm{mo}$ ) & 62 & 60 & 67 & 52 & \\
\hline Receptive anal $(>0 \%$ in past $12 \mathrm{mo})$ & 58 & 60 & 62 & 46 & \\
\hline Oral sex ( $>0 \%$ in past $12 \mathrm{mo}$ ) & 93 & 94 & 93 & 94 & \\
\hline Intoxicated sex ( $>0 \%$ in past $12 \mathrm{mo})$ & 64 & 63 & 66 & 61 & \\
\hline Vaginal sex ( $>0 \%$ in past $12 \mathrm{mo}$ ) & 37 & 35 & 35 & 44 & \\
\hline \multicolumn{6}{|l|}{ DSM-IV Psychiatric Disorder (\% lifetime) } \\
\hline Major depression & 36 & 36 & 35 & 40 & \\
\hline Bipolar I or II & 4 & 8 & 3 & 3 & \\
\hline Beck depression inventory $M(\mathrm{sd})$ & $12(9)$ & $11(8)$ & $13(10)$ & $10(9)$ & \\
\hline \multicolumn{6}{|l|}{ DSM-IV Substance Dependence (\% lifetime) } \\
\hline Sedative & 0 & 0 & 0 & 0 & \\
\hline Cannabis & 9 & 9 & 11 & 6 & \\
\hline Opioid & 0 & 0 & 0 & 0 & \\
\hline Cocaine & 7 & 7 & 5 & 14 & \\
\hline Hallucinogen & 0 & 0 & 0 & 0 & \\
\hline Alcohol & 17 & 15 & 20 & 14 & \\
\hline \multicolumn{6}{|l|}{ Methamphetamine Parameters } \\
\hline Methamphetamine dependent (\%) & 47 & 37 & 52 & 49 & \\
\hline Age at first METH use, yrs $M(\mathrm{sd})$ & $24(9)$ & $23(9)$ & $25(8)$ & $27(10)$ & \\
\hline Total METH use, yrs $M$ (sd) & $11(6)$ & $13(7)$ & $11(6)$ & $8(4)$ & $\mathrm{v} / \mathrm{v}>\mathrm{m} / \mathrm{m}^{*}$ \\
\hline Last use of METH, days Median (IQR) & $91(36,274)$ & $122(45,731)$ & $91(32,236)$ & $91(30,244)$ & \\
\hline \multicolumn{6}{|l|}{ HIV Parameters } \\
\hline HIV seropositive (\%) & 56 & 70 & 51 & 49 & $\mathrm{v} / \mathrm{v}>\mathrm{v} / \mathrm{m}, \mathrm{m} / \mathrm{m}^{*}$ \\
\hline HIV RNA, plasma (log copies/mL) $M(\mathrm{sd})$ & $2.1(1.9)$ & $2.4(1.7)$ & $2.0(2.0)$ & $1.7(1.9)$ & \\
\hline
\end{tabular}


TABLE 2: Multivariate linear regression coefficients for main, interaction, and stratified effects of executive functioning and COMT in seven sexual risk behavior models.

\begin{tabular}{|c|c|c|c|c|c|c|}
\hline \multirow[b]{3}{*}{$\begin{array}{l}\text { EF measure } \\
\text { Sexual Risk Behavior Model }\end{array}$} & \multicolumn{6}{|c|}{ Standardized Beta $^{(\mathrm{a})}$} \\
\hline & \multicolumn{2}{|c|}{ Main Effect } & \multirow{2}{*}{$\begin{array}{c}\text { Interaction } \\
\mathrm{EF} \times \mathrm{COMT} \\
\quad(n=192)\end{array}$} & \multicolumn{3}{|c|}{ Straitfied $^{(\mathrm{b})}$} \\
\hline & $\begin{array}{c}\mathrm{EF} \\
(n=192)\end{array}$ & $\begin{array}{c}\text { COMT } \\
(n=192)\end{array}$ & & $\begin{array}{l}\mathrm{Val} / \mathrm{Val} \\
(n=54)\end{array}$ & $\begin{array}{l}\text { Val/Met } \\
(n=103)\end{array}$ & $\begin{array}{l}\text { Met/Met } \\
(n=35)\end{array}$ \\
\hline \multicolumn{7}{|l|}{ Domain Deficit Score } \\
\hline (1) Partners (\# past $12 \mathrm{mo}$ ) & $0.21^{* *}$ & 0.10 & $0.50^{* *}$ & 0.03 & $0.20^{* *}$ & $0.52^{* * *}$ \\
\hline (2) Condom use (\% past $12 \mathrm{mo}$ ) & 0.03 & 0.13 & 0.24 & - & - & - \\
\hline (3) Insertive anal (\% past $12 \mathrm{mo}$ ) & 0.06 & 0.07 & $0.50^{* *}$ & -0.18 & 0.11 & 0.18 \\
\hline (4) Receptive anal (\% past $12 \mathrm{mo}$ ) & 0.05 & 0.05 & $0.44^{*}$ & -0.17 & 0.11 & 0.13 \\
\hline (5) Oral sex (\% past $12 \mathrm{mo})$ & -0.10 & 0.07 & 0.40 & - & - & - \\
\hline (6) Intoxicated sex (\% past $12 \mathrm{mo}$ ) & 0.07 & -0.06 & 0.08 & - & - & - \\
\hline (7) Vaginal sex (\% past $12 \mathrm{mo}$ ) & -0.03 & -0.04 & -0.28 & - & - & - \\
\hline \multicolumn{7}{|l|}{ Wisconsin Card Sort Test (T-score) } \\
\hline (1) Partners (\# past $12 \mathrm{mo}$ ) & -0.09 & 0.08 & -0.18 & - & - & - \\
\hline (2) Condom use (\% past $12 \mathrm{mo}$ ) & -0.10 & 0.12 & -0.25 & - & - & - \\
\hline (3) Insertive anal (\% past $12 \mathrm{mo}$ ) & 0.01 & 0.07 & -0.31 & - & - & - \\
\hline (4) Receptive anal (\% past $12 \mathrm{mo}$ ) & -0.02 & 0.04 & -0.38 & - & - & - \\
\hline (5) Oral sex (\% past $12 \mathrm{mo})$ & $0.20^{* *}$ & 0.09 & 0.13 & - & - & - \\
\hline (6) Intoxicated sex (\% past $12 \mathrm{mo}$ ) & -0.04 & -0.07 & 0.32 & - & - & - \\
\hline (7) Vaginal sex (\% past $12 \mathrm{mo}$ ) & 0.04 & -0.04 & -0.04 & - & - & - \\
\hline \multicolumn{7}{|l|}{ Trails B (T-score) } \\
\hline (1) Partners (\# past $12 \mathrm{mo}$ ) & -0.01 & 0.08 & -0.54 & - & - & - \\
\hline (2) Condom use (\% past $12 \mathrm{mo}$ ) & -0.07 & 0.11 & 0.03 & - & - & - \\
\hline (3) Insertive anal (\% past $12 \mathrm{mo}$ ) & -0.06 & 0.06 & $-0.99 * *$ & 0.18 & -0.03 & $-0.38^{* *}$ \\
\hline (4) Receptive anal (\% past $12 \mathrm{mo}$ ) & -0.04 & 0.03 & $-0.75^{*}$ & 0.17 & -0.06 & -0.22 \\
\hline (5) Oral sex (\% past $12 \mathrm{mo})$ & 0.10 & 0.06 & $-0.68^{*}$ & $0.35^{* *}$ & 0.01 & 0.01 \\
\hline (6) Intoxicated sex (\% past $12 \mathrm{mo}$ ) & -0.11 & -0.07 & 0.13 & - & - & - \\
\hline (7) Vaginal sex (\% past $12 \mathrm{mo}$ ) & 0.07 & -0.04 & 0.37 & - & - & - \\
\hline \multicolumn{7}{|l|}{ Halstead Category Test (T-score) } \\
\hline (1) Partners (\# past $12 \mathrm{mo}$ ) & -0.11 & 0.08 & -0.16 & - & - & - \\
\hline (2) Condom use (\% past $12 \mathrm{mo}$ ) & $-0.16^{* *}$ & 0.11 & $-1.13^{* *}$ & 0.08 & $-0.19^{*}$ & $-0.49^{* * *}$ \\
\hline (3) Insertive anal (\% past $12 \mathrm{mo}$ ) & 0.01 & 0.05 & -0.22 & - & - & - \\
\hline (4) Receptive anal (\% past $12 \mathrm{mo}$ ) & -0.01 & 0.03 & -0.59 & - & - & - \\
\hline (5) Oral sex (\% past $12 \mathrm{mo})$ & 0.06 & 0.06 & -0.50 & - & - & - \\
\hline (6) Intoxicated sex (\% past $12 \mathrm{mo}$ ) & -0.04 & -0.06 & -0.44 & - & - & - \\
\hline (7) Vaginal sex (\% past $12 \mathrm{mo}$ ) & -0.05 & -0.04 & 0.25 & - & - & - \\
\hline
\end{tabular}

(a) all regression models adjusted for METH status, HIV status, age at first intercourse.

(b) stratified analysis of EF effects by genotype was conducted if a significant $(P<.10)$ interaction was observed.

EF: executive functioning; COMT: catechol-O-methyltransferease $(0=\mathrm{Val} / \mathrm{Val} ; 1=\mathrm{Val} / \mathrm{Met} ; 2=\mathrm{Met} / \mathrm{Met})$

${ }^{*} P<.10 ; * * P<.05, * * * P<.005$.

dysfunction and sexual risk behavior among an AfricanAmerican sample of men and women poly-substance abusers with and without HIV infection. However, three major methodological differences may explain our discordant findings. First, Gonzalez et al. [7] estimated sexual risk behavior in the past 6 months compared to our window of 12 months and also utilized a composite score rather than individual sexual risk behaviors as their dependent variable.
Second, executive dysfunction was assessed using the Iowa Gambling Task, delayed non-matching to sample paradigm, and Stroop task-reaction time version which, respectively, measure decision-making, working memory, and response inhibition. Although these tests are well justified, other components of executive functioning such as perseveration, cognitive sequencing, and concept formation which were assessed in the current study, were not examined. Third 


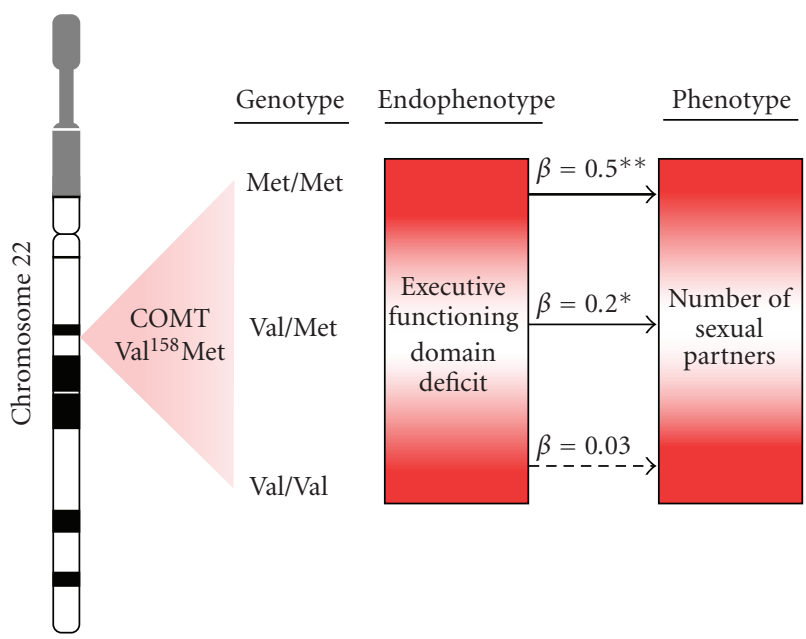

Figure 1: A theoretical model illustrating the interaction effect of a single genotype (i.e., COMT Val158Met polymorphism) and endophenotype (i.e., executive functioning domain deficit) on a behavioral phenotype (i.e., number of sexual partners). Among carriers of the COMT Met/Met and to a lesser extent the Val/Met genotype the association between executive functioning deficit and number of sexual partners is significantly stronger than among carriers of the Val/Val genotype. ${ }^{*} P<.05$; ${ }^{* *} P<.01$.

and finally, regression models were adjusted for sensation seeking, a factor shown in previous research to be associated with sexual risk behavior [34-37]; however, in the current study sensation seeking data was not available and was not adjusted for. Thus, future work examining the association between executive dysfunction and sexual risk behaviors are warranted; particularly research utilizing larger samples with diverse measures of executive functioning and models adjusting for sensation seeking and other personality covariates.

Novel to the current study, we demonstrated several genotype (i.e., COMT) by endophenotype (i.e., executive dysfunction) interactions for sexual risk behaviors. A relaxed significance criterion $(P<.10)$ produced significant interactions for number of sexual partners, condom use, insertive and receptive anal sex, as well as oral sex. These interactions collectively advocate for further investigation of genotypeendophenotype interactions for sexual risk behavior. However, due to the exploratory nature of these interactions our discussion will be confined to interactions observed for number of sexual partners, frequency of insertive anal sex and condom use, as interactions observed in these models met the traditional significance criterion $(P<.05)$.

We observed both a main and interaction effect for number of sexual partners, albeit only within the model including the composite executive functioning deficit score. In this model we found that among carriers of the Met allele (i.e., Met/Met or Val/Met), a positive association between executive functioning deficit and number of sexual partners was present. Thus, among Met allele carriers those with greater deficit scores reported greater number of sexual partners; whereas among Val/Val carriers this association was not significant. Similar to results for number of sexual partners, stratified analysis showed that among carriers of the Met/Met but not Val/Met or Val/Val genotype an positive association between executive dysfunction and frequency of insertive anal sex was present, although only statistically significant for models including the Trails B test. Thus, individuals with lower $T$-scores (i.e., greater impairment) on Trails B reported greater frequency of insertive anal sex only if they were carriers of the Met/Met genotype. Finally, the strongest interaction observed was between COMT and the Halstead Category Test for frequency of condom use. Contrary to the expected association, results suggest a negative association among carriers of the Met/Met genotype in which lower $T$-scores (i.e., greater impairment) on the Category Test was associated with an increased frequency of condom use. This unexpected finding may be a result of several factors. First, the psychometric properties of the questionnaire used to measure sexual risk behaviors in our study have not been reported and thus measurement error may be influencing our reported associations. Although there is no agreed upon "gold-standard" for measuring sexual risk behavior, recommendations from a review of 56 sexual risk behavior measures in the literature have been developed [38] and future studies should be encouraged to adopt these measurement strategies to improve accuracy of sexual risk behavior characterization. Second, recall deficits may result in sexual risk behavior reporting errors. This is particularly a concern when measuring sexual risk behavior retrospectively over large spans of time (i.e., 12 months) as was done in the current study. Post-hoc analysis within our sample showed no significant difference in recall deficit by COMT genotype, albeit there did appear to be a trend $\left(F_{(2,189)}=2.89 ; P=.058\right)$ in which carriers of the Val/Val genotype had greater deficits than that of Val/Met and Met/Met genotypes (data not shown). Thus, it is possible that recall deficits within the Val/Val group biased our findings toward those in the Met/Met group and should be interpreted with caution. Finally and most speculative, harm reduction campaigns have long aimed to increase condom use within both HIV-infected and METH using populations and our finding may be an artifact of their success.

Collectively, these findings provide a preliminary model of differential susceptibility to sexual risk behavior via executive dysfunction, dependent on COMT genotype, particularly the Met/Met genotype (Figure 1). Although the role of the Met/Met genotype is contrary to our hypothesis, our findings, when placed in the context of previous research are informative. Recent research has linked the COMT Met/Met genotype to novelty seeking behavior in healthy [39] and methamphetamine using [24] populations. In addition, work by Gonzalez et al. [7] on executive functioning and sexual risk behavior demonstrated that sensation seeking was independently associated with sexual risk, particularly among HIV-seropositive individuals. Thus, it appears that individuals with the Met/Met genotype may have a lower tolerance for monotony and may seek and participate in higher risk behaviors such as METH use or unprotected sex. Furthermore, work by our group and others [40] have suggested that possession of the Met allele enhances executive functioning in healthy controls; 
however, this neuroprotective effect is significantly reduced among individuals exposed to methamphetamine. Thus, it is probable that in our sample, of which approximately half were methamphetamine dependent, the putative protective effect of the Met/Met genotype is diminished and propensity to sexual risk behavior enhanced.

It is apparent that the associations between COMT, executive dysfunction, and sexual risk behavior are highly complex and context dependent. The current study provides preliminary evidence of these complex relationships and advocates for larger investigations that improve upon and consider several of the limitations that have been presented. Future work should also attempt to address independent and interaction effects of other putative polymorphisms particularly those involved in dopamine synthesis (e.g., Tyrosine Hydroxylase), metabolism (e.g., Monoamine Oxidase A), and reception (e.g., Dopamine Receptors D1-4). In addition, future transdisciplinary work that combines genetic and neurocognitive factors with psychosocial (e.g., trauma, stress) factors will provide valuable insights and elucidate a clearer picture of sexual risk behavior. Completion of such work in combination with the current as well as others previous work will further our understanding of the genotypic and endophenotypic factors involved in the phenotypic expression of sexual risk behaviors and potentially assist with risk identification, prevention, and treatment efforts in the future.

\section{HNRC Group}

The HNRC Group is affiliated with the University of California, San Diego, the Naval Hospital, San Diego, and the Veterans Affairs San Diego Healthcare System, and includes: Director: Igor Grant, M.D.; Codirectors: J. Hampton Atkinson, M.D., Ronald J. Ellis, M.D., Ph.D., and J. Allen McCutchan, M.D.; Center Manager: Thomas D. Marcotte, Ph.D.; Business Manager: Melanie Sherman; Naval Hospital San Diego: Braden R. Hale, M.D., M.P.H. (P.I.); Neuromedical Component: Ronald J. Ellis, M.D., Ph.D. (P.I.), J. Allen McCutchan, M.D., Scott Letendre, M.D., Edmund Capparelli, Pharm.D., Rachel Schrier, Ph.D.; Jennifer Marquie-Beck; Terry Alexander, R.N.; Neurobehavioral Component: Robert K. Heaton, Ph.D. (P.I.), Mariana Cherner, Ph.D., Steven Paul Woods, Psy.D., David J. Moore, Ph.D.; Matthew Dawson, Donald Franklin; Neuroimaging Component: Terry Jernigan, Ph.D. (P.I.), Christine Fennema-Notestine, Ph.D., Sarah L. Archibald, M.A., John Hesselink, M.D., Jacopo Annese, Ph.D., Michael J. Taylor, Ph.D., Neurobiology Component: Eliezer Masliah, M.D. (P.I.), Ian Everall, FRCPsych., FRCPath., Ph.D., Cristian Achim, M.D., Ph.D.; Neurovirology Component: Douglas Richman, M.D., (P.I.), David M. Smith, M.D.; International Component: J. Allen McCutchan, M.D., (P.I.); Developmental Component: Ian Everall, FRCPsych., FRCPath., Ph.D. (P.I.), Stuart Lipton, M.D., Ph.D.; Clinical Trials Component: J. Allen McCutchan, M.D., J. Hampton Atkinson, M.D., Ronald J. Ellis, M.D., Ph.D., Scott Letendre, M.D.; Participant Accrual and Retention Unit: J. Hampton Atkinson, M.D. (P.I.), Rodney von Jaeger, M.P.H.; Data Management Unit:
Anthony C. Gamst, Ph.D. (P.I.), Clint Cushman (Data Systems Manager), Daniel R. Masys, M.D. (Senior Consultant); Statistics Unit: Ian Abramson, Ph.D. (P.I.), Florin Vaida, Ph.D., Christopher Ake, Ph.D.

The views expressed in this paper are those of the authors and do not reflect the official policy or position of neither the Department of the Navy, Department of Defense, nor the United States Government.

\section{References}

[1] Joint United Nations Programme on HIV/AIDS, et al., "AIDS epidemic update, 86," 2006.

[2] R. J. Diclemente, C. P. Crittenden, E. Rose, et al., "Psychosocial predictors of HIV-associated sexual behaviors and the efficacy of prevention interventions in adolescents at-risk for HIV infection: what works and what doesn't work?" Psychosomatic Medicine, vol. 70, no. 5, pp. 598-605, 2008.

[3] J. D. Fortenberry, "Adolescent substance use and sexually transmitted diseases risk: a review," Journal of Adolescent Health, vol. 16, no. 4, pp. 304-308, 1995.

[4] B. C. Leigh and R. Stall, "Substance use and risky sexual behavior for exposure to HIV: issues in methodology, interpretation, and prevention," American Psychologist, vol. 48, no. 10, pp. 1035-1045, 1993.

[5] I. I. Gottesman and T. D. Gould, "The endophenotype concept in psychiatry: etymology and strategic intentions," American Journal of Psychiatry, vol. 160, no. 4, pp. 636-645, 2003.

[6] A. W. Stacy, M. D. Newcomb, and S. L. Ames, "Implicit cognition and HIV risk behavior," Journal of Behavioral Medicine, vol. 23, no. 5, pp. 475-499, 2000.

[7] R. Gonzalez, J. Vassileva, A. Bechara, et al., "The influence of executive functions, sensation seeking, and HIV serostatus on the risky sexual practices of substance-dependent individuals," Journal of the International Neuropsychological Society, vol. 11, no. 2, pp. 121-131, 2005.

[8] P. A. Hall, L. J. Elias, and M. Crossley, "Neurocognitive influences on health behavior in a community sample," Health Psychology, vol. 25, no. 6, pp. 778-782, 2006.

[9] E. D. Barker, J. R. Séguin, H. R. White, et al., "Developmental trajectories of male physical violence and theft: relations to neurocognitive performance," Archives of General Psychiatry, vol. 64 , no. 5, pp. 592-599, 2007.

[10] T. R. Barclay, C. H. Hinkin, S. A. Castellon, et al., "Ageassociated predictors of medication adherence in HIV-positive adults: health beliefs, self-efficacy, and neurocognitive status," Health Psychology, vol. 26, no. 1, pp. 40-49, 2007.

[11] E. W. Triche, N. Hossain, and M. J. Paidas, "Genetic influences on smoking cessation and relapse in pregnant women," Journal of Obstetrics and Gynaecology, vol. 28, no. 2, pp. 155-160, 2008.

[12] T. Rankinen and C. Bouchard, "Genetics of food intake and eating behavior phenotypes in humans," Annual Review of Nutrition, vol. 26, pp. 413-434, 2006.

[13] M. Tafti, S. Maret, and Y. Dauvilliers, "Genes for normal sleep and sleep disorders," Annals of Medicine, vol. 37, no. 8, pp. 580-589, 2005.

[14] M. R. Melis and A. Argiolas, "Dopamine and sexual behavior," Neuroscience and Biobehavioral Reviews, vol. 19, no. 1, pp. 1938, 1995.

[15] A. Nieoullon, "Dopamine and the regulation of cognition and attention," Progress in Neurobiology, vol. 67, no. 1, pp. 53-83, 2002. 
[16] J. M. Starr, H. Fox, S. E. Harris, I. J. Deary, and L. J. Whalley, "COMT genotype and cognitive ability: a longitudinal aging study," Neuroscience Letters, vol. 421, no. 1, pp. 57-61, 2007.

[17] S. W. Anderson and D. Tranel, "Neuropsychological consequences of dysfunction in human dorsolateral prefrontal cortex," in Handbook of Neuropsychology, J. Grafman, Ed., pp. 145-156, Elsevier, Amsterdam, The Netherlands, 2002.

[18] A. Bechara, "Risky business: emotion, decision-making, and addiction," Journal of Gambling Studies, vol. 19, no. 1, pp. 2351, 2003.

[19] D. A. Lewis, D. S. Melchitzky, S. R. Sesack, R. E. Whitehead, S. Auh, and A. Sampson, "Dopamine transporter immunoreactivity in monkey cerebral cortex: regional, laminar, and ultrastructural localization," Journal of Comparative Neurology, vol. 432, no. 1, pp. 119-136, 2001.

[20] J. Chen, B. K. Lipska, N. Halim, et al., "Functional analysis of genetic variation in catechol-O-methyltransferase (COMT): effects on mrna, protein, and enzyme activity in postmortem human brain," American Journal of Human Genetics, vol. 75, no. 5, pp. 807-821, 2004.

[21] J. H. Barnett, P. B. Jones, T. W. Robbins, and U. Müller, "Effects of the catechol-O-methyltransferase Val158Met polymorphism on executive function: a meta-analysis of the Wisconsin Card Sort Test in schizophrenia and healthy controls," Molecular Psychiatry, vol. 12, no. 5, pp. 502-509, 2007.

[22] S.-J. Tsai, C.-J. Hong, Y. W.-Y. Yu, and T.-J. Chen, "Association study of catechol-O-methyltransferase gene and dopamine D4 receptor gene polymorphisms and personality traits in healthy young Chinese females," Neuropsychobiology, vol. 50, no. 2, pp. 153-156, 2004.

[23] M. Reuter and J. Hennig, "Association of the functional catechol-O-methyltransferase VALI58MET polymorphism with the personality trait of extraversion," NeuroReport, vol. 16, no. 10, pp. 1135-1138, 2005.

[24] L. Hosák, J. Libiger, J. Cizek, M. Beránek, and E. Cermáková, "The COMT Val158Met polymorphism is associated with novelty seeking in Czech methamphetamine abusers: preliminary results," Neuroendocrinology Letters, vol. 27, no. 6, pp. 799-802, 2006.

[25] E. M. Drabant, A. R. Hariri, A. Meyer-Lindenberg, et al., "Catechol O-methyltransferase val158met genotype and neural mechanisms related to affective arousal and regulation," Archives of General Psychiatry, vol. 63, no. 12, pp. 1396-1406, 2006.

[26] L. McKusick, T. J. Coates, S. F. Morin, L. Pollack, and C. Hoff, "Longitudinal predictors of reductions in unprotected anal intercourse among gay men in San Francisco: the AIDS Behavioral Research Project," American Journal of Public Health, vol. 80, no. 8, pp. 978-983, 1990.

[27] A. T. Beck, Depression Causes and Treatment, University of Pennsylvania Press, Philadelphia, Pa, USA, 1972.

[28] R. K. Heaton and P. Staff, "Wisconsin Card Sorting Test: Computerized Version 2. Research Edition," 1993.

[29] N. A. DeFilippis and P. Staff, "Category Test: Computer Version," 1993.

[30] Army Individual Test Battery, Manual of Directions and Scoring, Adjunct General's Office, War Deparment, Washington, DC, USA, 1944.

[31] R. K. Heaton, I. Grant, N. Butters, et al., "The HNRC 500-neuropsychology of HIV infection at different disease stages. HIV Neurobehavioral Research Center," Journal of the International Neuropsychological Society, vol. 1, no. 3, pp. 231251, 1995.
[32] C. L. Carey, S. P. Woods, R. Gonzalez, et al., "Predictive validity of global deficit scores in detecting neuropsychological impairment in HIV infection," Journal of Clinical and Experimental Neuropsychology, vol. 26, no. 3, pp. 307-319, 2004.

[33] R. M. Baron and D. A. Kenny, "The moderator-mediator variable distinction in social psychological research. Conceptual, strategic, and statistical considerations," Journal of Personality and Social Psychology, vol. 51, no. 6, pp. 1173-1182, 1986.

[34] S. C. Kalichman, T. Heckman, and J. A. Kelly, "Sensation seeking as an explanation for the association between substance use and HIV-related risky sexual behavior," Archives of Sexual Behavior, vol. 25, no. 2, pp. 141-154, 1996.

[35] S. C. Kalichman, J. R. Johnson, V. Adair, D. Rompa, K. Multhauf, and J. A. Kelly, "Sexual sensation seeking: scale development and predicting AIDS-risk behavior among homosexually active men," Journal of Personality Assessment, vol. 62, no. 3, pp. 385-397, 1994.

[36] J. T. Parsons and P. N. Halkitis, "Sexual and drug-using practices of HIV-positive men who frequent public and commercial sex environments," AIDS Care, vol. 14, no. 6, pp. 815-826, 2002.

[37] C. S. Hendershot, S. A. Stoner, W. H. George, and J. Norris, "Alcohol use, expectancies, and sexual sensation seeking as correlates of HIV risk behavior in heterosexual young adults," Psychology of Addictive Behaviors, vol. 21, no. 3, pp. 365-372, 2007.

[38] S. M. Noar, C. Cole, and K. Carlyle, "Condom use measurement in 56 studies of sexual risk behavior: review and recommendations," Archives of Sexual Behavior, vol. 35, no. 3, pp. 327-345, 2006.

[39] V. E. Golimbet, M. V. Alfimova, I. K. Gritsenko, and R. P. Ebstein, "Relationship between dopamine system genes and extraversion and novelty seeking," Neuroscience and Behavioral Physiology, vol. 37, no. 6, pp. 601-606, 2007.

[40] V. S. Mattay, T. E. Goldberg, F. Fera, et al., "Catechol Omethyltransferase val158-met genotype and individual variation in the brain response to amphetamine," Proceedings of the National Academy of Sciences of the United States of America, vol. 100, no. 10, pp. 6186-6191, 2003. 


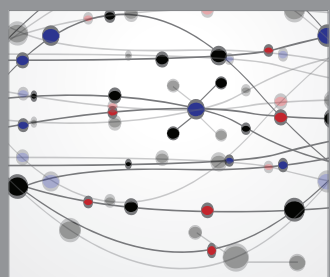

The Scientific World Journal
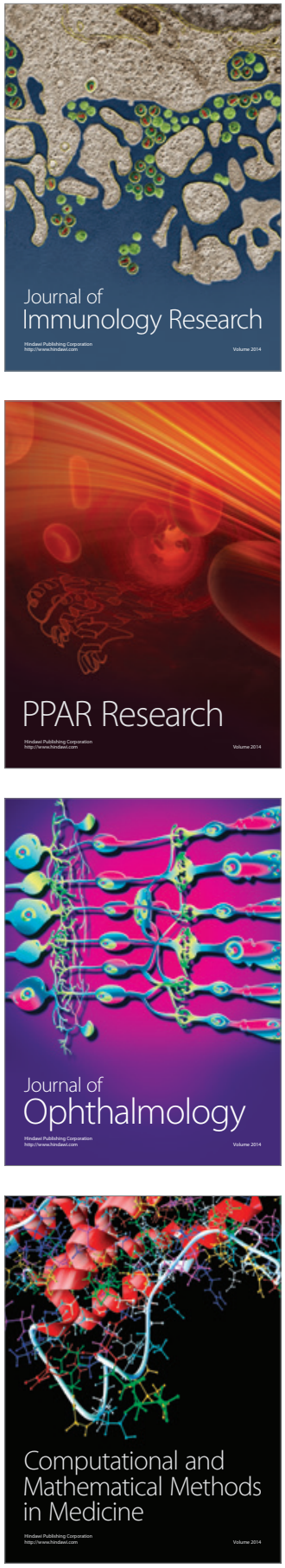

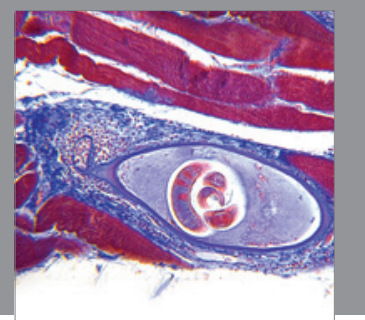

Gastroenterology

Research and Practice
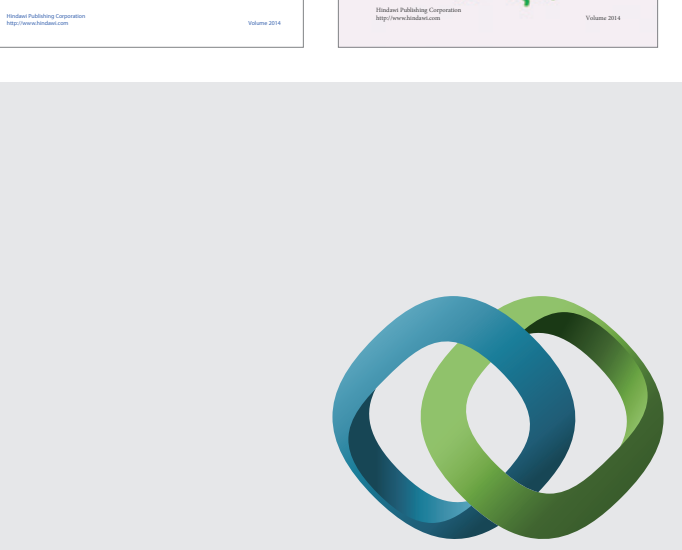

\section{Hindawi}

Submit your manuscripts at

http://www.hindawi.com
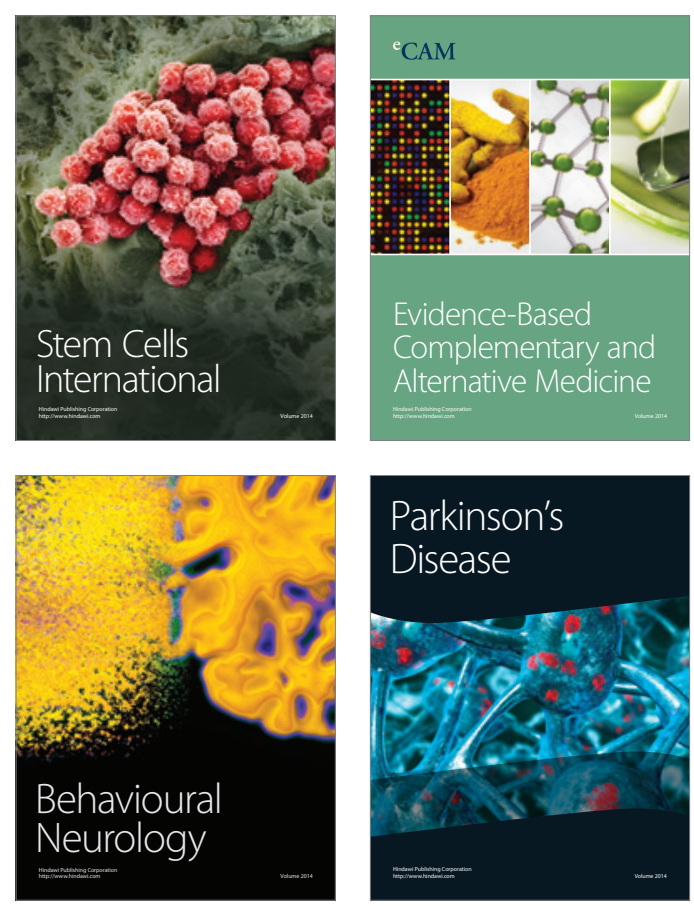

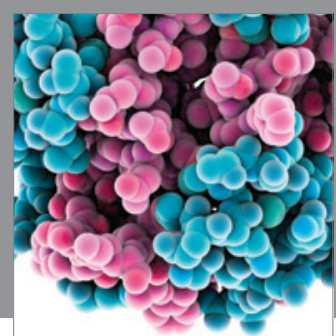

Journal of
Diabetes Research

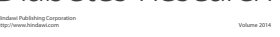

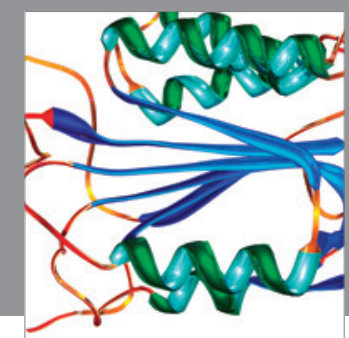

Disease Markers
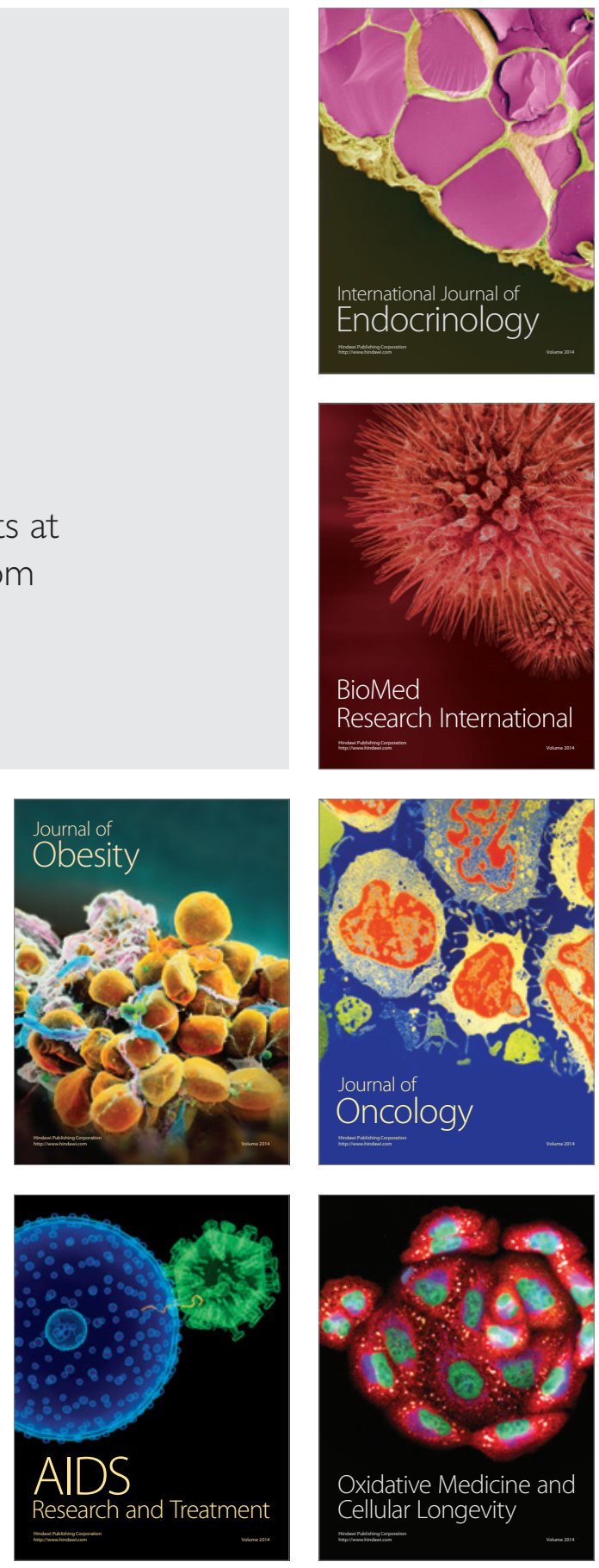